\title{
Maternal antibiotic use and child asthma: is the association causal?
}

\author{
Mingyu Zhang ${ }^{1}{ }^{1,2}$, Augusto A. Litonjua ${ }^{3}$ and Noel T. Mueller ${ }^{1,2}$
}

Affiliations: ${ }^{1}$ Dept of Epidemiology, Johns Hopkins Bloomberg School of Public Health, Baltimore, MD, USA. ${ }^{2}$ Welch Center for Prevention, Epidemiology and Clinical Research, Johns Hopkins University, Baltimore, MD, USA. ${ }^{3}$ Pediatric Pulmonary Division, Dept of Pediatrics, Golisano Children's Hospital at Strong, University of Rochester Medical Center, Rochester, NY, USA.

Correspondence: Noel T. Mueller, Welch Center for Prevention, Epidemiology and Clinical Research, Johns Hopkins University, 2024 E. Monument St, Baltimore, MD 21205, USA. E-mail: noeltmueller@jhu.edu

\section{@ERSpublications}

Maternal antibiotic use before, during and after pregnancy is associated with higher child asthma risk. Lack of specificity to the pregnancy period suggests the association is not causal or the window of susceptibility extends outside pregnancy. http://ow.ly/U29f30kFD2I

Cite this article as: Zhang M, Litonjua AA, Mueller NT. Maternal antibiotic use and child asthma: is the association causal? Eur Respir J 2018; 52: 1801007 [https://doi.org/10.1183/13993003.01007-2018].

Global antibiotic use has risen 65\% worldwide and 114\% in low- and middle-income countries between 2000 and 2015 [1]. During pregnancy, anywhere from $20 \%$ to over $40 \%$ of women may receive a course of antibiotics [2,3], and antibiotics account for $80 \%$ of medications used by pregnant women in the USA [4]. Although antibiotics in pregnancy can treat many infections, including bacterial vaginosis and urinary tract infections, they may also have unintended adverse consequences [4]. Given the ubiquitous exposure to antibiotics in pregnancy, even a small adverse effect of maternal antibiotic use on health outcomes in the mother or her child could pose a substantial public health problem.

Since the 1970s, the worldwide incidence and prevalence of asthma have increased markedly, with an estimated 334 million cases worldwide in 2014 [5, 6]. In children, asthma is the most common chronic disease, and for 5-14-year-olds, it is among the top 10 chronic conditions for disability-adjusted life years [5]. The multifactorial aetiology of child asthma begins with the mother. Maternal asthma, and exposure to cigarette smoke and air pollution in pregnancy are among the factors known to increase offspring risk for asthma [7, 8]. Preterm birth and low birth weight have also been associated with asthma risk [9]. More recently, several lines of investigation have provided intriguing evidence that perturbations to the natural mother-to-newborn transfer of microbial life may also play an important role in asthma pathogenesis.

Thanks to recent advances in high-throughput sequencing methods, we now know that most of our bodies' ecological niches are teeming with trillions of microbes. Antibiotic treatment can impact entire microbial communities, also known as microbiota, and the metabolites they produce for months or longer [10]. Interestingly, antibiotics taken in pregnancy have been associated not only with altered maternal microbiota but also with impacted infant microbiota [11, 12], suggesting antibiotics may affect fetal microbiota or metabolite exposure. Maternal microbiota and metabolites in utero may help train the fetal innate immune system and have been causally implicated in allergic airway disease in mice [13, 14]. When used at or after birth, antibiotics may disrupt mother-to-newborn transmission of healthy microbiota of the maternal gut, vagina, skin and/or breast milk $[15,16]$. In addition to effects on the microbiome and metabolome, antibiotics may alter epigenetics [17] and fetal development [18]. An important question is whether through these mechanistic associations, maternal-fetal exposure to antibiotics increases the risk of immune-mediated diseases, such as asthma. 
In the past several years, a burgeoning number of studies has examined the association between maternal antibiotic use during pregnancy and risk of child asthma and, to our knowledge, all have observed positive associations even after controlling for multiple potentially confounding factors [19-24]. But is this association causal? The two largest studies, which were based in national registries, concluded that this relationship may not be causal. Using data from 493785 Swedish children born in 2006-2010, ÖRTQVIST et al. [23] found that the association between prenatal antibiotics and child asthma disappeared in a sibling analysis. This finding suggested the link may be confounded by shared familial factors. In 910301 Danish children born in 1997-2010, Sтокноцм et al. [24] observed the association of maternal antibiotic use with offspring asthma was not specific to the pregnancy period (significant associations were also observed in the preconception and postnatal periods) and it was stronger for antibiotics prescribed for respiratory tract infections. Based on these findings, they concluded that the association may be due to maternal propensity for infection rather than antibiotics, per se.

In this issue of the European Respiratory Journal, LoEwen et al. [25] provide fresh evidence that maternal antibiotic exposure is associated with a dose-dependent increase in child asthma risk. They examined data from a population-based cohort study of 213661 mother-child dyads in Manitoba, Canada, from 1996 to 2012. Almost all classes of antibiotics were associated with varying risk of asthma, and the associations persisted after adjusting for multiple confounders and were consistent by delivery method, child sex and infant feeding method. The overall association also did not appear to differ by trimester of pregnancy. However, similar to the study by Sтокноцм et al. [24], the investigators did not find that the association was unique to the pregnancy period.

LOEwEN et al. [25] interpreted their finding, that maternal antibiotic use in the periods before, during and after pregnancy was associated with childhood asthma, as evidence that antibiotics taken in pregnancy, specifically, may not be causally associated with childhood asthma. They offered alternative hypotheses for their findings. First, they posited that the association may be due to a genetic susceptibility to infections inherited by offspring that imparts a predisposition for asthma. If genetic susceptibility for infections were associated with asthma, and heritable, one would expect it to be associated with asthma in the mother. Yet, when the authors adjusted for maternal asthma, the association was not attenuated, thus undermining this hypothesis. The authors also conjectured that maternal vitamin D deficiency could explain the association. Vitamin D is involved in development of the fetal immune system and lungs, and low vitamin D has been associated with higher risk of infections in pregnancy [26] and offspring asthma [27]. Vitamin D supplementation has been shown to reduce risk of infections and, nonsignificantly, antibiotic use [28]. There is also evidence from two trials that vitamin D supplementation in pregnancy lowers risk of child asthma or recurrent wheeze by $25 \%$ [29]. Unfortunately, the current study did not have data on maternal vitamin D status; thus, future research is needed to test the hypothesis that vitamin D status may play a role in the association of maternal antibiotic use and child asthma.

There are alternative explanations for the findings of LoEwEn et al. [25]. Antibiotics substantially alter the composition of microbiota and their metabolite production. Some of these changes disappear within weeks or months of stopping antibiotic treatment; other impacts may last indefinitely [10]. Thus, antibiotics taken preconception may have residual effects on the maternal microbiota during pregnancy, which has been associated with long-term risk of asthma in children [30]. Antibiotics taken by the mother post partum may perturb mother-to-newborn transmission of microbiota vis-à-vis impacts on microbial communities in the maternal skin or breast milk [31]. Because of these viable alternative explanations, we agree with the authors' conclusion that their results "do not firmly support nor refute a directly causal pregnancy-specific relationship".

An interesting observation, which was not explained by the authors, is that the rural population, which constituted nearly half of the study sample, had higher rates of antibiotic use, yet considerably lower incidence of asthma (7.6 versus 12.4 cases per 1000 person-years). To our knowledge, this observation is novel and has not been reported in prior studies of antibiotics and asthma. One might speculate that this observation could be due to some rural populations having greater exposure to diverse microbial communities that may make them more resilient against the risk of asthma associated with antibiotics. Unfortunately, the authors did not directly examine whether the association of maternal antibiotics with child asthma was modified by rural versus urban residence in their study, but this should be explored in future studies.

Several factors strengthened this study. The large sample size, made possible by use of comprehensive administrative health data from Manitoba, allowed the authors to conduct a series of subgroup analyses adjusted for or stratified by important variables. The authors were limited, however, by not having information on maternal or childhood infections, maternal vitamin D status, or intrapartum antibiotics among other factors. Also, because they had data from almost the entire province of Manitoba, there was 
minimal chance of biased selection into the study (e.g. self-selection or nonresponse) or out of the study (e.g. differential loss to follow-up). Recall bias was also unlikely, as covariates such as antibiotic use and maternal asthma were extracted from administrative databases rather than by participant questionnaire. Another strength of the study, although not novel, was the authors' ability to leverage maternal antibiotic data 9 months before and after pregnancy to determine if the association was specific to the pregnancy period.

The decision of whether or not to prescribe antibiotics in pregnancy is a delicate balancing act. On one hand, antibiotics treat bacterial infections and in many cases, avoiding these medications may be more harmful than taking them, as untreated infections may lead to serious maternal and fetal complications. On the other hand, antibiotic resistance and the potential risk of antibiotics on offspring health outcomes should also be considered. As with other clinicians, obstetricians should heed recommendations for judicious administration of pre- and perinatal antibiotics [32], given that $30 \%$ of antibiotic prescriptions may be unnecessary [33] and antibiotic misuse poses a public health threat by generating antibiotic-resistant bacteria. Moreover, whether or not maternal antibiotics are causally associated with child asthma, clinicians should keep in mind they may be "treating for two" [34], given associations of some antibiotics with fetal growth [18], birth defects [35] and other child health outcomes.

In conclusion, LoEwen et al. [25] should be commended for their rigorous examination of the association between maternal antibiotics and risk of childhood asthma in a large population-based study. Although the authors observed evidence for a biological gradient (dose-response association), they did not identify a particular window of susceptibility; thus, limiting their causal inference about the pregnancy-specific effects of antibiotics. Their study still does not rule out the possibility that maternal antibiotics taken before, during or after pregnancy are associated with offspring risk of asthma. More research is needed to understand the life course of antibiotic effects on the maternal vaginal, gut and breast milk microbiota and their metabolites, as well as to better understand possible rural-urban differences in the association of maternal antibiotics with child asthma. As even the most rigorous observational study cannot prove or disprove causality [36], a more conclusive approach to addressing this question might be to take advantage of previous randomised trials of prenatal antibiotics [37,38], following up the offspring and measuring biomarkers and incidence of asthma.

Conflict of interest: A.A. Litonjua reports receiving author royalties from UpToDate, Inc., and consultancy fees from AstraZeneca, LP, outside the submitted work.

Support Statement: N.T. Mueller is supported by the National Heart, Lung, And Blood Institute of the National Institutes of Health under award number K01HL141589, and by grants from the Mid-Atlantic Nutrition Obesity Research Center (P30DK072488) and the Foundation for Gender Specific Medicine. The content is solely the responsibility of the authors and does not necessarily represent the official views of the National Institutes of Health. Funding information for this article has been deposited with the Crossref Funder Registry.

\section{References}

1 Klein EY, Van Boeckel TP, Martinez EM, et al. Global increase and geographic convergence in antibiotic consumption between 2000 and 2015. Proc Natl Acad Sci USA 2018; 115: E3463-E3470.

2 Broe A, Pottegard A, Lamont RF, et al. Increasing use of antibiotics in pregnancy during the period 2000-2010: prevalence, timing, category, and demographics. BJOG 2014; 121: 988-996.

3 de Jonge L, Bos HJ, van Langen IM, et al. Antibiotics prescribed before, during and after pregnancy in the Netherlands: a drug utilization study. Pharmacoepidemiol Drug Saf 2014; 23: 60-68.

4 Bookstaver PB, Bland CM, Griffin B, et al. A review of antibiotic use in pregnancy. Pharmacotherapy 2015; 35: 1052-1062.

5 Asher I, Pearce N. Global burden of asthma among children. Int J Tuberc Lung Dis 2014; 18: 1269-1278.

6 Croisant S. Epidemiology of asthma: prevalence and burden of disease. Adv Exp Med Biol 2014; 795: 17-29.

7 Lavigne É, Bélair MA, Rodriguez Duque D, et al. Effect modification of perinatal exposure to air pollution and childhood asthma incidence. Eur Respir J 2018; 51: 1701884.

8 Ferrante G, Antona R, Malizia V, et al. Smoke exposure as a risk factor for asthma in childhood: a review of current evidence. Allergy Asthma Proc 2014; 35: 454-461.

9 Sonnenschein-van der Voort AM, Arends LR, de Jongste JC, et al. Preterm birth, infant weight gain, and childhood asthma risk: a meta-analysis of 147,000 European children. J Allergy Clin Immunol 2014; 133: 1317-1329.

10 Willing BP, Russell SL, Finlay BB. Shifting the balance: antibiotic effects on host-microbiota mutualism. Nat Rev Microbiol 2011; 9: 233-243.

11 Zou ZH, Liu D, Li HD, et al. Prenatal and postnatal antibiotic exposure influences the gut microbiota of preterm infants in neonatal intensive care units. Ann Clin Microbiol Antimicrob 2018; 17: 9.

12 Chernikova DA, Koestler DC, Hoen AG, et al. Fetal exposures and perinatal influences on the stool microbiota of premature infants. J Matern Fetal Neonatal Med 2016; 29: 99-105.

13 Vuillermin PJ, Macia L, Nanan R, et al. The maternal microbiome during pregnancy and allergic disease in the offspring. Semin Immunopathol 2017; 39: 669-675.

14 Thorburn AN, McKenzie CI, Shen S, et al. Evidence that asthma is a developmental origin disease influenced by maternal diet and bacterial metabolites. Nat Commun 2015; 6: 7320. 
15 Walker RW, Clemente JC, Peter I, et al. The prenatal gut microbiome: are we colonized with bacteria in utero? Pediatr Obes 2017; 12: Suppl. 1, 3-17.

16 Mueller NT, Bakacs E, Combellick J, et al. The infant microbiome development: mom matters. Trends Mol Med 2015; 21: 109-117.

17 Vidal AC, Murphy SK, Murtha AP, et al. Associations between antibiotic exposure during pregnancy, birth weight and aberrant methylation at imprinted genes among offspring. Int J Obes (Lond) 2013; 37: 907-913.

18 Mueller NT, Rifas-Shiman SL, Blaser MJ, et al. Association of prenatal antibiotics with foetal size and cord blood leptin and adiponectin. Pediatr Obes 2017; 12: 129-136.

19 Lapin B, Piorkowski J, Ownby D, et al. Relationship between prenatal antibiotic use and asthma in at-risk children. Ann Allergy Asthma Immunol 2015; 114: 203-207.

20 Metsala J, Lundqvist A, Virta LJ, et al. Prenatal and post-natal exposure to antibiotics and risk of asthma in childhood. Clin Exp Allergy 2015; 45: 137-145.

21 Mulder B, Pouwels KB, Schuiling-Veninga CC, et al. Antibiotic use during pregnancy and asthma in preschool children: the influence of confounding. Clin Exp Allergy 2016; 46: 1214-1226.

22 Stensballe LG, Simonsen J, Jensen SM, et al. Use of antibiotics during pregnancy increases the risk of asthma in early childhood. J Pediatr 2013; 162: 832-838.

23 Örtqvist AK, Lundholm C, Kieler H, et al. Antibiotics in fetal and early life and subsequent childhood asthma: nationwide population based study with sibling analysis. BMJ 2014; 349: g6979.

24 Stokholm J, Sevelsted A, Bonnelykke K, et al. Maternal propensity for infections and risk of childhood asthma: a registry-based cohort study. Lancet Respir Med 2014; 2: 631-637.

25 Loewen K, Monchka B, Mahmud SM, et al. Prenatal antibiotic exposure and childhood asthma: a population-based study. Eur Respir J 2018; 52: 1702070.

26 Akoh CC, Pressman EK, Cooper E, et al. Low vitamin D is associated with infections and proinflammatory cytokines during pregnancy. Reprod Sci 2018; 25: 414-423.

27 Chawes BL, Bonnelykke K, Jensen PF, et al. Cord blood 25(OH)-vitamin D deficiency and childhood asthma, allergy and eczema: the COPSAC2000 birth cohort study. PLoS One 2014; 9: e99856.

28 Tran B, Armstrong BK, Ebeling PR, et al. Effect of vitamin D supplementation on antibiotic use: a randomized controlled trial. Am J Clin Nutr 2014; 99: 156-161.

29 Wolsk HM, Chawes BL, Litonjua AA, et al. Prenatal vitamin D supplementation reduces risk of asthma/recurrent wheeze in early childhood: A combined analysis of two randomized controlled trials. PLoS One 2017; 12: e0186657.

30 Benn CS, Thorsen P, Jensen JS, et al. Maternal vaginal microflora during pregnancy and the risk of asthma hospitalization and use of antiasthma medication in early childhood. J Allergy Clin Immunol 2002; 110: 72-77.

31 Soto A, Martin V, Jimenez E, et al. Lactobacilli and bifidobacteria in human breast milk: influence of antibiotherapy and other host and clinical factors. J Pediatr Gastroenterol Nutr 2014; 59: 78-88.

32 McNulty C. Management and treatment of common infections. Antibiotic guidance for primary care: For consultation and local adaptation. https:/assets.publishing.service.gov.uk/government/uploads/system/uploads/ attachment_data/file/664740/Managing_common_infections_guidance_for_consultation_and_adaptation.pdf Date last updated: October 2017. Date last accessed: May 292018.

33 Fleming-Dutra KE, Hersh AL, Shapiro DJ, et al. Prevalence of inappropriate antibiotic prescriptions among US ambulatory care visits, 2010-2011. JAMA 2016; 315: 1864-1873.

34 Centers for Disease Control and Prevention. Treating for Two: Medicine and Pregnancy. https://www.cdc.gov/ ncbddd/birthdefects/documents/ncbddd_birth-defects_medicationuseonepager_cdcrole.pdf Date last updated: 2018. Date last accessed: May 292018.

35 ACOG Committee Opinion No. 494: Sulfonamides, nitrofurantoin, and risk of birth defects. Obstet Gynecol 2011; 117: 1484-1485.

36 Ejima K, Li P, Smith DL Jr, et al. Observational research rigour alone does not justify causal inference. Eur J Clin Invest 2016; 46: 985-993.

37 Andrews WW, Sibai BM, Thom EA, et al. Randomized clinical trial of metronidazole plus erythromycin to prevent spontaneous preterm delivery in fetal fibronectin-positive women. Obstet Gynecol 2003; 101: 847-855.

38 Carey JC, Klebanoff MA, Hauth JC, et al. Metronidazole to prevent preterm delivery in pregnant women with asymptomatic bacterial vaginosis. National Institute of Child Health and Human Development Network of Maternal-Fetal Medicine Units. N Engl J Med 2000; 342: 534-540. 